percentage and fat mass as assessed by bioelectrical impedance analysis, are more strongly related to risk is unknown and has not been explored in any prospective study. In addition, the independent role of body fat distribution in the development of endometrial cancer remains unclear.

Methods We analysed data from 202,796 women in UK Biobank, aged 40-69 years at study entry. Trained personnel collected data on body size and composition. Cox proportional hazard models, with time in study as the underlying time metric, were used to estimate hazard ratios (HR) and corresponding 95\% confidence intervals (CIs) for the association of body size and body composition with risk of endometrial cancer. Analyses were stratified by year of birth and year of recruitment and adjusted for age, socio-economic deprivation score, age at menarche, menopausal status and age at menopause, use of oral contraceptive and hormone replacement therapy, diabetes status, physical activity and smoking status. Models that assessed the association of fat distribution measures and fat-free mass on cancer risk were additionally adjusted for BMI in order to examine their independent effects.

Results During a mean follow up of 5.5 years, 651 incident cases of endometrial cancer were identified, with a mean age of diagnosis of 63.1 years. The HR per standard deviation increase in BMI was $1.64(1.53-1.75)$ and was comparable to that of body fat percentage $(\mathrm{HR}=1.73,95 \% \mathrm{CI}: 1.56-1.90)$ and fat mass $(\mathrm{HR}=1.64,95 \% \mathrm{CI}: 1.53-1.76)$; however, in terms of model fit assessed by likelihood ratio chi square statistics, BMI appeared to be the most informative measure. All measures of fat distribution (waist and hip circumference, waist to hip ratio, waist to height ratio and trunk fat percentage) also showed strong positive associations with endometrial cancer risk (Ptrend <0.001); however, these associations were substantially attenuated after adjusting for BMI (Ptrend $>0.10$ ). Fat-free mass, a marker of muscle and bone mass, was also strongly associated with endometrial cancer risk $(\mathrm{HR}=1.58$, 95\% CI:1.47-1.69), and the association persisted after adjusting for BMI (HR=1.15, 95\%CI: 1.03-1.29).

Conclusion The results of this study support the continued use of BMI in assessing the risk of endometrial cancer associated with obesity.

\section{OP34 ENDURING PSYCHOLOGICAL IMPACT OF CHILDHOOD CANCER ON SURVIVORS AND THEIR FAMILIES IN IRELAND: A NATIONAL QUALITATIVE STUDY}

\footnotetext{
1,2PM Barrett*, 'L Mullen, ${ }^{1} \mathrm{~T}$ McCarthy. ${ }^{1}$ National Cancer Control Programme, 200 Parnell Street, Dublin, Ireland; ${ }^{2}$ Irish Clinical Academic Training (ICAT) Programme, School of Public Health, University College Cork, Ireland
}

\subsection{6/jech-2019-SSMabstracts.34}

Background Five-year survival after childhood cancer has improved in Ireland in recent decades and is currently $81 \%$. There are thousands of adult survivors of childhood cancer (ASCC) living in Ireland, but little is known about their psychological and social needs. Ireland's National Cancer Strategy 2017-2026 highlighted the need to establish the psycho-social needs of ASCC. This study aims to establish the major expressed psycho-social needs of this group for the first time. Methods Seven focus groups were conducted with ASCC and parents in Dublin, Cork and Galway in 2018. Focus groups were promoted through social media, print media, radio, voluntary organisations, and clinical networks. Survivors were invited to participate if they were diagnosed with cancer before age 18. Braun \& Clarke's Framework for Thematic Analysis was used to identify semantic and latent themes. Member-check was undertaken to confirm that participants' views and experiences were accurately recorded.

Results The 33 participants (15 ASCC, 18 parents) had experienced a range of haematological and solid tumours in childhood. Five overarching themes were generated: (1) Enduring psychological impact on survivors; Many ASCC experience high levels of psychological distress as adults, including delayed trauma which can precipitate mental health crises in adulthood. (2) Enduring psychological impact on family members; Parents and siblings have unmet psychological needs relating to their experience of childhood cancer. (3) Enduring impact on family dynamics; ASCC and parents expressed high levels of fear and guilt relating to their cancer experience, which impacted on their family interactions. (4) Challenges accessing support; Psychological support services are inadequate to meet the needs of ASCC and their families, and are often only available through private providers. ASCC are not routinely offered psychological support during follow-up. (5) Desired model of care; No single service model appeals to all ASCC, and flexibility is required in the delivery of psychological support (individual vs. group, formal vs. informal).

Conclusion ASCC and their family members experience enduring psychological effects relating to their diagnosis and treatment. Psychological support services are inadequate to meet the needs of this growing population in Ireland. The study was limited by its participants who were self-selected, majority female, and may have experienced psychological distress regardless of their previous experience of cancer. The findings are being used to guide development of enhanced psychooncology services in the Irish public healthcare system, in line with the National Cancer Strategy.

\section{OP35 A SYSTEMATIC REVIEW OF POPULATION-BASED QUALITY INDICATORS FOR END OF LIFE CANCER CARE}

${ }^{1}$ LA Henson*, ${ }^{2} \mathrm{P}$ Edmonds, ${ }^{1} \mathrm{H}$ Johnson, ${ }^{1} \mathrm{~A}$ Johnston, ${ }^{3} \mathrm{CNY}$ Ling, ${ }^{3} \mathrm{~A}$ Sklavounos, ${ }^{1} \mathrm{C}$ EllisSmith, 'W Gao. ${ }^{1}$ Cicely Saunders Institute of Palliative Care, Policy and Rehabilitation, King's College London, London, UK; 'ㄹepartment of Palliative Care, King's College Hospital NHS Foundation Trust, London, UK; ${ }^{3} G K T$ School of Medical Education, King's College London, London, UK

\subsection{6/jech-2019-SSMabstracts.35}

Background Improving the quality of cancer care throughout the disease trajectory is an international priority. Populationbased quality indicators (QIs) are key to this process yet remain predominantly used for evaluating care during the early, often curative, stages of disease.

Aim To identify all existing QIs for cancer patients with advanced disease and/or at the end-of-life, and to evaluate each indicator's measurement properties and appropriateness for use. Methods We searched five electronic databases from inception to February 2019 for studies describing the development, review and/or testing of QIs for adults with advanced cancer and/or at the end-of-life. For each QI identified we extracted descriptive information (numerator; denominator; benchmarking data; care domain) and assessed six measurement properties (acceptability; evidence-base; definition; feasibility; reliability; validity). Assessments were based on previously established criteria with four possible ratings: positive; intermediate; negative; unknown. Ratings were collated, and each QI classified as either: appropriate for use; inappropriate for use; or, of limited 
testing. Among the QIs determined appropriate for use we generated a further shortlist by excluding those that were specific to certain patient sub-groups and/or care settings. The shortlist was further reduced by identifying similar/related QIs and retaining the indicator with the highest rating.

Results Our search yielded 7,231 references. Following screening, 35 references met our eligibility criteria and were included in the review. We identified 288 QIs for patients with advanced cancer and/or at the end-of-life. Most evaluated physical aspects of care $(n=103,35.8 \%)$ or structure and processes of care $(n=109,37.8 \%)$. There was a limited number of QIs relevant to psychosocial $(n=18)$ or spiritual/cultural $(n=3)$ care domains. $27.8 \%(n=80)$ of QIs were assessed as appropriate for use; $40.3 \%(n=116)$ inappropriate for use, and $31.9 \%(n=92)$ had limited testing. Acceptability and validity were the measurement properties with the fewest positive assessments (13.2\% and $21.9 \%$ respectively). Only 16 QIs $(5.6 \%)$ reported any benchmarking data. Our shortlist comprised 36 QIs after those specific to patient sub-groups or care settings were excluded. This was further reduced to 15 once duplicate and/or related QIs were removed.

Conclusion Only a small proportion of QIs developed for patients with advanced cancer and/or at the end-of-life have received adequate testing and/or are appropriate for use. Further testing is recommended, particularly with regards to acceptability and validity, as well as research to establish benchmarking data and to expand QIs relevant to psychosocial, cultural and spiritual care domains. To support cancer services in conducting comprehensive and meaningful assessments of quality, we propose 15 QIs, identified from our review as being scientifically sound, applicable across care settings and which collectively evaluate quality across multiple domains of care.

\section{Nutrition/Obesity}

\section{OP36 TRANS FATTY ACID BIOMARKERS AND INCIDENT TYPE 2 DIABETES: POOLED ANALYSIS OF 10 PROSPECTIVE COHORT STUDIES IN THE FATTY ACIDS AND OUTCOMES RESEARCH CONSORTIUM (FORCE)}

\begin{abstract}
${ }^{1} \mathrm{HTM} \mathrm{Lai}^{*},{ }^{2} \mathrm{~F}$ Imamura, ${ }^{3} \mathrm{AV}$ Ardisson Korat, ${ }^{4} \mathrm{R}$ Murphy, ${ }^{5} \mathrm{NL}$ Tintle, ${ }^{6}$ J Bassett, ${ }^{7} \mathrm{~J}$ Chen, ${ }^{8}$ K Kroeger, ${ }^{2} \mathrm{NG}$ Forouhi, ${ }^{8} \mathrm{MB}$ Schulze, ${ }^{9} \mathrm{WS}$ Harris, ${ }^{10} \mathrm{RS}$ Vasan, ${ }^{3} \mathrm{FB}$ Hu, ${ }^{13} \mathrm{RN}$ Lemaitre, ${ }^{6} \mathrm{GG}$ Giles, ${ }^{7} \mathrm{~L}$ Djousse, ${ }^{11} \mathrm{I}$ Brouwer, ${ }^{12} \mathrm{JHY}$ Wu, ${ }^{12} \mathrm{M}$ Marklund, ${ }^{1} \mathrm{R}$ Micha, ${ }^{13} \mathrm{RN}$ Lemaitre. ${ }^{1}$ Friedman School of Nutrition Science and Policy, Tufts University, Boston, USA; ${ }^{2}$ MRC Epidemiology Unit, University of Cambridge, Cambridge, UK; ${ }^{3}$ Department of Nutrition, Harvard School of Public Health, Harvard University, Boston, USA; ${ }^{4}$ Centre of Excellence in Cancer Prevention, School of Population and Public Health, The University of British Columbia, Vancouver, Canada; ${ }^{5}$ Department of Mathematics and Statistics, Dordt College, Sioux Center, USA; ${ }^{6}$ Cancer Epidemiology and Intelligence Division, Cancer Council Victoria, Melbourne, Australia; 'Division of Aging, Brigham and Women's Hospital, Boston, USA; ${ }^{8}$ Department of Molecular Epidemiology, German Institute of Human Nutrition, Nuthetal, Germany; ${ }^{9}$ Department of Internal Medicine, Sanford School of Medicine, University of South Dakota, Sioux Falls, USA; ${ }^{10}$ Boston University School of Medicine, Boston University, Boston, USA; ${ }^{11}$ Department of Health Sciences, Faculty of Science, and Amsterdam Public Health Research, Institute, Vrije Universiteit Amsterdam, Amsterdam Netherlands; ${ }^{12}$ The George Institute for Global Health, Faculty of Medicine, University of New South Wales, Sydney, Australia; ${ }^{13}$ Department of Medicine, Cardiovascular Health Research Unit, University of Washington, Seattle, USA
\end{abstract}

\subsection{6/jech-2019-SSMabstracts.36}

Background Type 2 diabetes (T2D) is a major risk factor associated with cardiometabolic diseases, and a major contributor towards mortality and morbidity, given its rapidly rising prevalence worldwide. In experimental studies, trans-fatty acids (TFAs) exert harmful biologic effects that may affect T2D risk, but findings from observational studies remain inconclusive, especially for biomarkers which provide an objective advantage with less recall bias and estimation errors. By pooling multiple studies, we may also increase generalizability, statistical power, and address potential interactions by subgroups. Therefore, we assessed prospective associations between circulating biomarkers of individual TFAs and incident T2D in a large, diverse sample.

Methods We pooled ten prospective cohort or nested-case-control studies from Australia, Germany, Iceland, UK, and the USA to perform an analysis using harmonized individual level data for TFA biomarkers and incident T2D. Fatty acids (FAs) were measured in plasma phospholipid, red blood cell membrane phospholipid, or total plasma collected between 19902008 from 22,711 participants aged $\geq 18$ years without prevalent diabetes. Evaluated TFAs included trans-16:1n-9, sum of trans-18:1 isomers (trans-18:1n6 to trans-18:1n12), sum of trans-18:2 isomers (cis/trans-18:2, trans/cis-18:2, trans/trans18:2), and individual trans-18:2 isomers. The multivariableadjusted relative risk or odds ratio was estimated for each cohort by lipid compartments using a pre-specified protocol for definitions of exposures, covariates, and outcomes for statistical analysis. Association estimates were pooled using fixedeffects inverse-variance weighted meta-analysis.

Results During an average maximum of 14 years of follow-up, 2,244 cases of incident T2D were identified. Median levels of TFAs across cohorts were $0.05-0.18 \%$ total FAs for trans$16: 1 \mathrm{n}-9, \quad 0.09-2.05 \%$ for total trans-18:1, $0.10-0.73 \%$ for total trans-18:2, and $0.01-0.36 \%$ for individual trans-18:2 isomers. In overall pooled analysis, TFAs evaluated per interquintile range were not significantly associated with risk of T2D. Relative risks for individual TFAs were $1.02(0.78-1.32)$ for trans-16:1n-9, $0.92(0.79-1.08)$ for total trans-18:1, 1.16 (0.98-1.37) for trans/trans-18:2, 0.98 (0.79-1.21) for cis/ trans-18:2, $0.93(0.76-1.14)$ for trans/cis-18:2, and 0.90 (0.78-1.04) for total trans-18:2. Findings were consistent when TFAs were assessed categorically by study-specific quintiles, and when associations were pooled within lipid compartment (phospholipids or total plasma).

Conclusion We found that biomarker levels of TFAs were not significantly associated with risk of incident T2D in this international pooling project. Findings may reflect no effect of circulating TFA on T2D or be influenced by mixed TFA sources (industrial or ruminant), or to a general decline in TFA exposure during this period. Associations with T2D for higher levels of TFA biomarkers should be investigated.

\section{OP37 HEREDITARY HAEMOCHROMATOSIS: ASSOCIATIONS WITH MORBIDITY AND IRON SUPPLEMENT USE IN 451,243 UK BIOBANK PARTICIPANTS}

JL Atkins*, LC Pilling, D Melzer. Epidemiology and Public Health Research Group, College of Medicine and Health, University of Exeter, Exeter, UK

\subsection{6/jech-2019-SSMabstracts.37}

Background Hereditary haemochromatosis $(\mathrm{HH})$ is the most common and probably the most treatable genetic disorder in Europe, but many patients are misdiagnosed or diagnosed too late. $\mathrm{HH}$ causes iron overload and is predominantly due to the HFE p.C282Y genetic variant. $\mathrm{HH}$ is easily prevented and 